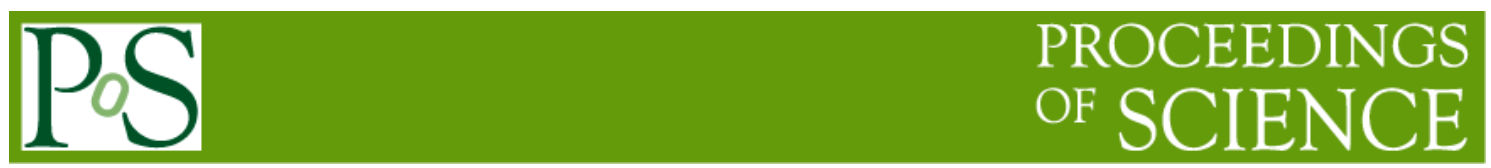

Aspects of the quest for gravitational waves

\title{
E. Coccia
}

Gran Sasso Science Institute, INFN; and Università di Roma "Tor Vergata".

Viale F. Crispi 7, L’Aquila

E-mail: eugenio.coccia@gssi.infn.it

Direct detection of gravitational waves from astrophysical sources is one of the great challenges of contemporary experimental physics. Gravitational waves were predicted almost 100 years ago by Einstein and their detection motivates today about one thousand scientists, constructing new apparatuses and developing advanced technologies and data analysis algorithms. Observation and study of gravitational radiation will give unique information on compact cosmic objects (black holes and neutron stars) and on gravitational physics at extreme conditions. The study of primordial gravitational waves would uniquely allow the investigation of processes in the very early universe, since gravitons decoupled from the primordial plasma below the Planck scale and hence are able to bring information on very high energy physics which cannot be accessed experimentally in any other way.

The status of this field of research is reviewed, and the interest of particular signals is discussed.

XV Workshop on Neutrino Telescopes

March 11-15, 2013

Venice, Italy 
1. Introduction

Gravitational waves (GWs) were predicted by Einstein as a consequence of his general theory of relativity [1]. They can be emitted by a variety of extreme astrophysical systems and propagate at the speed of light as ripples in the geometry of spacetime.

The first decade of the $21^{\text {st }}$ century has seen a shift in the technologies used in gravitational wave searches as the first generation of large gravitational wave interferometers has begun operation at their design sensitivities, taking up the baton from the bar detectors that pioneered the search for the first direct detection of gravitational waves $[2,3,4]$.

LIGO, Virgo and GEO 600 [5] are a network of interferometric detectors aiming to make the first direct observations of GWs. They consist of two experimental sites in the US (Hanford, WA and Livingston, LA) and two in Europe (Hannover, Germany and Cascina, Italy). An additional experimental site to host an underground cryogenic detector named KAGRA (previously known as LCGT [6]) is under construction in Japan while a proposal to build a third LIGO site in India is currently being evaluated[7].

These detectors use kilometer-scale Michelson interferometry to measure the fractional differential change $\mathrm{dL} / \mathrm{L}$ in the distances of two orthogonally positioned pairs of masses[8]. Such changes are expected to occur upon the passage of a GW. The fractional distance change $\mathrm{dL} / \mathrm{L}$ between two such pairs of perpendicularly arranged masses defines $h(t)$, the GW strain as measured by the detectors. This is a direct measure of the strength of the local spacetime distortions (which is the GW itself) folded with coefficients of order unity that depend on the direction of the wave source and the orientation of the interferometer [9].

The network of GW detectors is currently (2013) undergoing upgrades which are expected to produce a factor of about 10 improvement in sensitivity [10,11]. These will be the advanced LIGO and advanced Virgo detectors and are expected to come online, respectively, in 2015 and 2016.

We are then on the threshold of a new era of gravitational wave astrophysics. First generation interferometers have broken new ground in sensitivity and have proven technique, second generation detectors are starting installation and will expand the "Science" (astrophysics) by factor of 1000. So it is a good time to look to the potentiality of these detectors for astrophysics, cosmology and particle physics.

Data from gravitational wave detectors are searched for many types of possible signals. In particular signals from compact binary coalescences (CBC), including Binary Star systems. The gravitational waveform from a binary neutron star coalescence is well modelled and matched filtering can be used to search for signals and measure the system parameters. In the era of advanced detectors, the LSC and Virgo will search in near real-time for CBC and burst signals for the purpose of rapidly identifying event candidates. It is likely that the first detected signal will be from a $\mathrm{CBC}$.

I report here on the relevance of these detectors discussing other three particular GW signals: the relic GW background from the very early Universe (section 2), the burst signal from instabilities in compact stars (section 3) and the burst signal from a supernova scenario, in which much can be understood by a simultaneous detection of the GW and neutrino bursts (section 4). In the last section, the perspectives of the quest for gravitational waves are summarized.

\section{Relic GW background}

The energy range between the grand unification scale $\mathrm{M}_{\mathrm{GUT}} \sim 10^{16} \mathrm{GeV}$ and the Planck scale $\mathrm{M}_{\mathrm{Pl}}$ $\simeq 1.22 \times 10^{19} \mathrm{GeV}$ is crucial for fundamental physical questions and for testing current ideas about grand unification, quantum gravity, string theory.

There are important experimental results in particle physics that can be translated into statements about this energy range, like the accurate measurement of Gauge coupling constant versus energy at 
accelerators and the measurement of proton lifetime. Here we take a cosmological point of view: informations on this energy range can only come from particles which decoupled from the primordial plasma at very early time $[12,13]$. Particles which stay in thermal equilibrium down to a decoupling temperature $\mathrm{T}_{\mathrm{dec}}$ can only carry information on the state of the Universe at $\mathrm{E} \sim \mathrm{T}_{\mathrm{dec}}$. All information on physics at higher energies has in fact been obliterated by the successive interactions.

The condition for thermal equilibrium is that the rate $\mathrm{G}$ of the processes that maintain equilibrium be larger than the rate of expansion of the Universe, as measured by the Hubble parameter $H$ [14]. The rate is given by $\mathrm{G}=\mathrm{n \sigma}|\mathrm{v}|$ where $\mathrm{n}$ is the number density of the particle in question, and for massless or light particles in equilibrium at a temperature $\mathrm{T}, \mathrm{n} \sim \mathrm{T}^{3} ;|\mathrm{V}| \sim 1$ is the typical velocity and $\sigma$ is the crosssection of the process. Consider for instance the weakly interacting neutrinos. In this case the equilibrium is mantained, e.g., by electron-neutrino scattering, and at energies below the $\mathrm{W}$ mass $\sigma \sim \mathrm{G}_{\mathrm{F}}^{2}<\mathrm{E}^{2}>\sim$ $\mathrm{G}_{\mathrm{F}}^{2} \mathrm{~T}^{2}$, where $\mathrm{G}_{\mathrm{F}}$ is the Fermi constant and $\left\langle\mathrm{E}^{2}>\right.$ is the average energy squared. The Hubble parameter during the radiation dominated era is related to the temperature by $\mathrm{H} \sim \mathrm{T}^{2} / \mathrm{M}_{\mathrm{Pl}}$.

Therefore

$$
\left(\frac{\Gamma}{H}\right)_{\text {neutrino }}=\frac{G_{F}^{2} T^{5}}{T^{2} / M_{P l}} \approx\left(\frac{T}{1 M e V}\right)^{3}
$$

Even the weakly interacting neutrinos, therefore, cannot carry information on the state of the Universe at temperatures larger than approximately $1 \mathrm{MeV}$. If we repeat the above computation for gravitons, the Fermi constant $\mathrm{G}_{\mathrm{F}}$ is replaced by Newton constant $\mathrm{G}=1 / \mathrm{M}_{\mathrm{Pl}}^{2}$ ( using units $\mathrm{i}=c=k_{B}$ ) and at energies below the Planck mass

$$
\left(\frac{\Gamma}{H}\right)_{\text {graviton }} \sim\left(\frac{T}{M_{P l}}\right)^{3}
$$

The gravitons are therefore decoupled below the Planck scale. At the Planck scale the above estimate of the cross section is not valid and nothing can be said without a quantum theory of gravity. It follows that relic gravitational waves are a potential source of information on very high-energy physics. Gravitational waves produced in the very early Universe have not lost memory of the conditions in which they have been produced, as it happened to all other particles, but still retain in their spectrum, typical frequency and intensity, important information on the state of the very early Universe, and therefore on physics at correspondingly high energies, which cannot be accessed experimentally in any other way. It is also clear that the property of gravitational waves that makes them so interesting, i.e. their extremely small cross section, is also responsible for the difficulties of the experimental detection.

Incidentally, thinking in terms of cross-sections, one is lead to ask how comes that gravitons could be detectable, since the graviton-matter cross section is smaller than the neutrino-matter cross section, at energies below the $\mathrm{W}$-mass, by a factor $\mathrm{G}^{2} / \mathrm{G}_{\mathrm{F}}^{2} \sim 10^{-67}$ and neutrinos are already so difficult to detect. The answer is that gravitons are bosons, and therefore their occupation number per cell of phase space can be $n_{k} \gg 1$; in interesting cases, in the relic stochastic background we can have $n_{k} \sim 10^{40}$ or larger, and the squared amplitude for exciting a given mode of the detector grows as $\mathrm{n}_{\mathrm{k}}^{2}$. So, we should not speak of detecting gravitons, but rather classical gravitational waves. Neutrinos, in contrast, are fermions and for them $\mathrm{n}_{\mathrm{k}} \leq 1$. 
There are various production mechanisms from which we might expect cosmological gravitational waves, including cosmic strings [15], amplification of vacuum fluctuations following inflation [16, 17], pre-Big-Bang models [18, 19], or the electroweak phase transition [20].

Whatever the production mechanism, the intensity of a stochastic background of gravitational waves (GWs) can be characterized by the dimensionless quantity

$$
\Omega_{g w}(f)=\frac{f}{\rho_{c}} \frac{d \rho_{g w}}{d f}
$$

where $d \rho_{\mathrm{gw}}$ is the energy density of gravitational radiation contained in the frequency range $f$ to $\mathrm{f}+\mathrm{df}$ and $\rho_{\mathrm{c}}$ is the present value of the critical energy density for closing the Universe [11]. In terms of the present value of the Hubble constant $\mathrm{H}_{\mathrm{o}}$, the critical density is given by

$$
\rho_{c}=\frac{3 H_{o}^{2}}{8 \pi G}
$$

The value of $H_{o}$ is usually written as $H_{o}=h_{o} \times 100 \mathrm{~km} /(\mathrm{s} \mathrm{Mpc})$, where $h_{o}$ parametrizes the existing experimental uncertainty.

As a signal is expected to be much smaller than current detector noise, and because we assume both the detector noise and the signal to be Gaussian random variables, it is not feasible to distinguish the two in a single detector. We must therefore search for the stochastic GW background using two or more detectors. The optimal method is to cross-correlate the strain data from a pair, or several pairs of detectors [21].

There are also mechanisms, such as due to magnetars or rotating neutron stars which can give a component to the stochastic background in term of a superposition of unresolvable gravitational-wave signals of astrophysical origin.

Direct limits on the amplitude of the stochastic gravitational-wave background using the data from a two-year science run of the Laser Interferometer Gravitational-wave Observatory (LIGO) [22] have been reported in 2009. These results constrained the energy density of the stochastic gravitational-wave background normalized by the critical energy density of the universe, in the frequency band around 100 $\mathrm{Hz}$, to be less than $6.9 \times 10^{-6}$ at $95 \%$ confidence. These data ruled out models of early universe evolution with relatively large equation-of-state parameter [23], as well as cosmic (super)string models with relatively small string tension [24] that are favoured in some string theory models [25]. This search for the stochastic background improved upon the indirect limits from the Big Bang Nucleosynthesis and cosmic microwave background at $100 \mathrm{~Hz}$.

New results were presented in 2012 with the first joint search for a stochastic background using data from the LIGO and Virgo interferometers [26]. In a frequency band of $600-1000 \mathrm{~Hz}$, a $95 \%$ upper limit on the amplitude of $\Omega_{\mathrm{GW}}(\mathrm{f})=0.33(\mathrm{f} / 900 \mathrm{~Hz})^{3}$. These new limits are a factor of seven better than the previous best in this frequency band.

The conclusion is that LIGO and Virgo can explore the Universe at temperatures $\mathrm{T} \sim 10^{7} \mathrm{GeV}$.

\section{Compact stars}

Neutron stars (NS) are the final state of stars which, after the exhaustion of their nuclear fuel and the subsequent explosion and ejection of the external layers, remain with a core more massive than the 
Chandrasekhar mass $\mathrm{M}_{\mathrm{Ch}} \simeq 1.4 \mathrm{M}_{\odot}$ (but still the core must be lighter than a critical value $\mathrm{M}_{\mathrm{bh}}<\mathrm{O}(2-$ 3) $\mathrm{M}_{\odot}$, beyond which a black hole instead forms). When $\mathrm{M}_{\mathrm{Ch}}<\mathrm{M}_{\text {core }}<\mathrm{M}_{\mathrm{bh}}$, the core of the star collapses under its own weight, until it reaches a radius $\mathrm{R} \simeq 10 \mathrm{~km}$, where the self-gravity of the star is now balanced by the neutron degeneracy pressure. As a consequence, the nuclear matter inside the star is compressed to extreme densities [27].

The internal structure of neutron stars depends strongly on the equation of state; however in the inner core, say $\mathrm{R}<\mathrm{O}(1) \mathrm{km}$, the density reaches values of order $1 \mathrm{GeV} / \mathrm{fm}^{3}$. We are therefore in a regime governed by QCD at high density.

Neutron star perturbations can be described in terms of quasinormal modes, which can be excited by many possible mechanisms: accreting material, crust breaking, starquakes, onset of phase transitions, coalescence, etc. These perturbations can excite, in particular, the neutron star quasinormal modes described by spherical harmonics with $1=2$, which dominates the emission of GWs. Furthermore, if the NS is rotating, even the radial oscillation will induce a time varying quadrupole moment, and in this case energy will be liberated in GWs.

The frequency of the quasinormal mode depends, in a calculable way, on the equation of state in the NS interior. Therefore the value of the GW frequency carries important information on the internal NS structure.

It must be remarked that for GWs even an object such as the core of a NS is basically transparent, because of the smallness of gravitational cross-sections. Therefore, GWs generated inside the core, for instance as a consequence of a corequake, travel unaffected outside the NS. This is of course very different from electromagnetic waves, for which the NS interior is totally opaque, and is an excellent example of the fact that GW astronomy can potentially open up a completely new window on the Universe, unaccessible to electromagnetic observations.

A NS accreting matter at a steady rate from a companion can undergo periodically some structural changes which are accompanied by the emission of bursts of GWs. The magnetic field of the NS is not sufficiently strong to channel the accreting matter toward the poles, and therefore the accretion is spherically symmetric. Each time a layer of about $1 \mathrm{~m}$ of material is accreted (which happens in a time which, depending on the particular star, can be between a few hours and a few days) a thermonuclear flash takes place, and is observed as an x-ray burst. These sources therefore repeatedly emit x-ray bursts.

The mass radius relation of a NS depends on the equation of state, but it is always such that the larger is the mass, the smaller is the radius. Thus, when a NS accretes material, its new equilibrium radius decreases. If the NS were a fluid, a continuous accretion of matter would produce a continuous decrease of the radius. However, neutron stars have a solid crust, about $1 \mathrm{~km}$ thick and with a rigidity which, in the inner part, is huge by terrestrial standards. Therefore the radius will rather stays constant until sufficient material has been accreted so that the crust can be broken, and the evolution of the radius will rather be a sequence of jumps. In each jump a certain amount of energy is released. The important issue, for our purposes, is that the amount of energy released per jump, radiated away in GWs, depends on the composition of the NS and on the high energy physics holding in such compact objects.

The maximum energy that can be liberated in a single starquake is [28]:

$$
\Delta E_{r a d} \sim 10^{-9} M_{\odot} c^{2}\left(\frac{R}{14 k m}\right)\left(\frac{M_{\odot}}{M}\right)\left(\frac{\theta_{\text {max }}}{10^{-3}}\right)
$$

We have taken $14 \mathrm{~km}$ as a reference value for $\mathrm{R}$, since this is the typical value of the radius for a fast rotating NS in a large range of masses. $\mathrm{q}_{\max }$ is the maximum strain angle that the crust can sustain without breaking. 
Another possibility, giving possibly larger GW emissions, is that the starquake due to the crust breaking, rather than being the source of the GWs, might be the trigger for some more important structural changes inside the NS. In particular, the core of a NS can perform a phase transition from a hadronic to a deconfined quark-gluon phase. In the literature [30,31] it has been considered the possibility that an accreting NS acquires a sufficient mass to perform completely the transition from a hadronic core to a quark-gluon core. The energy gained in the transition, for a NS with a mass $\mathrm{M}=1.5 \mathrm{M}$, is of order $0.15 \mathrm{Mc}^{2}$, and it has been observed that this large energy can excite quasinormal modes and be liberated in a GW bursts (with high efficiency if the NS rotates sufficiently fast). Particularly interesting appear the discontinuity g-modes [29], having typical frequency in the range $0.5-1.4 \mathrm{kHz}$ and constituting an unique probe for density discontinuities, like the ones induced by phase transitions. One can imagine that the phase transition does not take place suddenly and completely in the whole core. Rather, each time a critical mass is accreted, a starquake takes place and transforms successive layers of the NS core from the hadronic to the deconfined phase. In this case the 0.15 solar masses will not be released in a single, very large bursts, but rather in a series of bursts. One can imagine variants of this scenario in which the phase transition involves strange quark matter, hybrid quark stars, or a phase of color crystallization. In all cases, unfortunately, a computation of the energy liberated by the phase transition in a NS layer as a consequence of the starquake appears a rather difficult task.

Another possible realization of a GW source is provided by the r-mode instability in strange stars (see Chapter 12 of Ref. [27] for an introduction to strange stars). As discussed in Ref. [33], in stars made of strange quark matter the r-mode instability has a dynamics quite distinct from the neutron star case. In particular, in an accreting strange star the evolution of the GW amplitude generated by the r-mode during its first year of evolution consists of a repeated series of bursts on a time scales from hours to months (see, in particular, Fig. 4 of Ref. [33]). The GW amplitude has been estimated to be

$$
h \sim 2 \times 10^{-20} \alpha\left(\frac{1 k p c}{r}\right)\left(\frac{M}{1.4 M_{\odot}}\right)\left(\frac{1 m s}{P_{\text {rot }}}\right)^{3}\left(\frac{R}{10 k m}\right)^{3}
$$

where $\mathrm{P}_{\text {rot }}$ is the rotation period and $\mathrm{a}$ is the r-mode amplitude. During the first year of evolution of a young strange star the parameter a performs large oscillations from very low values, of the order of $10^{-15}$, up to values of order one. These rapid variations therefore result in a series of GW bursts.

The main feature of this source, is that within the model discussed in Ref. [33] this kind of activity can take place only for very young quark stars, i.e., in their first year of evolution.

The detection and study of these sources are a challenge for the next generation of interferometers.

\section{The Supernova}

A supernova is an explosion of a massive supergiant star. No events in nature surpasses its raw power: about $10^{53} \mathrm{erg} \mathrm{s}^{-1}$ (equivalent to $10^{46} \mathrm{~J} \mathrm{~s}^{-1}$ ) is released as neutrinos from a 'core-collapse' supernova, which is as much instantaneous power as all the rest of the luminous, visible Universe combined. They give birth to the most exotic states of matter known: neutron stars and black holes. Supernovae have been at the forefront of astronomical research for the better part of a century, and yet no one is sure how they work [34].

We discuss here the scientific motivations for a joint analysis of GWs and low-energy neutrino data to probe the processes powering a supernova explosion [35].

Several mechanisms in a core-collapse supernova can give rise to GW bursts. Signals may last from milliseconds to seconds. While the astrophysical motivation for expecting GWs to accompany core- 
collapse supernovae is strong, the expected rate, GW strength and waveform morphology are uncertain. The expected energy going into GWs would be $10^{-10}-10^{-4}$ of a solar mass, or $10^{44}-10^{50}$ ergs. This is only a small fraction of the energy liberated in neutrinos: the core collapse of a massive star is expected to produces a huge flux of neutrinos. Actually nearly all (about 99\%) of the binding energy $\mathrm{O}\left(10^{53} \mathrm{ergs}\right)$ of the resulting neutron star (or black hole) ends up in neutrinos escaping on a short timescale (a few tens of seconds). The neutrinos (and antineutrinos) produced are of all flavours, and have energies in the few to tens of MeV range. The burst of neutrinos from SN1987A in the Large Magellanic Cloud, detected by various neutrino detectors, confirmed the baseline model of stellar collapse. On the contrary, the estimates of GW bursts associated with supernova rely on models. Most such models are not yet threedimensional, do not incorporate the entire set of possibly relevant physics, and do not predict robust supernova explosions as observed in the electromagnetic universe. So, despite the availability of multiple potential explosion mechanisms and their associated multi-dimensional dynamics and GW signatures, the current picture is unlikely to be complete.

It is well worth to study the supernova signals with GWs and neutrino detectors with a multimessenger approach.

The first important point is that GWs and neutrinos from core-collapse supernovae are emitted in the inner-most, high density region of the supernova core which cannot be probed electromagnetically. GWs and neutrinos thus are the only messengers that can carry "live" dynamical information from deep inside a dying massive star and constrain the detailed, yet unknown, mechanism driving the core-collapse supernova explosion. Another point is that both GWs and neutrinos leave the core-collapse event on approximately the same time scale (seconds) in contrast to electromagnetic radiation, which can take hours or days to become visible to astronomers. Moreover, a visible supernova near the edge of sensitivity may be a rather poor tag of core collapse for both GW and neutrino detectors separately, in the presence of detector background, given what will likely be a large uncertainty on the occurrence itself of a collapse and on its timing [36]. Let me develop these considerations.

Several of the world's present neutrino detectors are sensitive to a neutrino burst from a galactic supernova. Super-K, a 50-kton water Cherenkov detector in Japan, would observe some $\sim 8000$ events for a core collapse at the center of the Milky Way, $2.5 \mathrm{kpc}$ away [37].

The LVD [38] and Borexino [39] scintillation detectors at Gran Sasso in Italy, and KamLAND [40] in Japan, would observe hundreds of interactions. The IceCube detector at the South Pole, although nominally a multi-GeV neutrino detector, would observe a coincident increase in count rate in its phototubes due to a diffuse burst of Cherenkov photons in the ice, and has sensitivity to a galactic supernova.

The duty factor of neutrino detectors in observing mode has typically been $90 \%$ or larger.

The Super-K, LVD, IceCube and Borexino detectors are also operating as part of the SNEWS (SuperNova Early Warning System) network [41,42], which has the aim of providing a prompt alert to astronomers in the case of a coincident supernova neutrino burst.

Uncertainties on the rate of supernovae in the nearby universe are significant. According to current rate estimates, there is about one supernova every 40 years in the galaxy, 3-4 supernovae per century in the local group (at a distance of $\sim 1 \mathrm{Mpc}$ ), about one supernova every other year between 3-5 Mpc, with an integral rate of one supernova per year out to the Virgo cluster. These rates are more likely to be lower than upper bounds.

A significant fraction of galactic or very close extragalactic supernovae (within $1 \sim \mathrm{Mpc}$ ) could be optically silent, not only because of no or very weak explosions, but also because of dust extinction of the electromagnetic (optical) emission. We can use GWs and neutrinos to identify such core-collapse events that do not lead to a strong electromagnetic display. A neutrino and GW coincidence, in the absence of a strong prompt electromagnetic signal, would provide smoking-gun evidence for this weak or "failing" 
supernova scenario. It is worth noting that the rate of such events may be comparable to the rate of supernovae with optical signals. An example is SN 2008iz that exploded in M82 in late January 2008, but was discovered serendipitously in the electromagnetic spectrum (through radio observations) only more than a year later. Besides the two large spiral galaxies M31 (Andromeda) and the Milky Way, there are a number of smaller galaxies in the Local Group that may have a much higher core-collapse rate than presently expected. These are the Magellanic Clouds (SN1987A exploded in the LMC) and IC 10, IC 1613, and NGC 6822 (all within $\sim 50-700 \mathrm{kpc}$ ). Combining GW data with neutrino observations would enhance the ability to find electromagnetically dark or obscured supernovae in our nearby universe.

There is an assured improvement offered to the core-collapse supernova reach by the joint analysis of the GW and neutrino detectors. This is due to the possibility of a tighter search window and lower background rate. Estimates have been done and are in progress in order to quantify this improvement. Let me report two examples from ref. [35].

1) In the all-sky GW burst search using the first-year data of LIGO's fifth science run, a false alarm rate of 1 in 100 years in the $64-200 \mathrm{~Hz}$ frequency band corresponds to a three-detector network signal-tonoise ratio (SNR) threshold of 8.5. For frequencies above $200 \mathrm{~Hz}$, the corresponding SNR threshold is lower, but the interferometers' strain sensitivity is lower at this frequency band. Requiring a coincidence of GW events within $\mathrm{O}(1 \mathrm{~s})$ of a neutrino signal tuned at a rate of 1 per day would allow $\mathrm{GW}$ detectors to operate at a false alarm rate of $3 \times 10^{-5} \mathrm{~Hz}$, which in turn corresponds to a SNR threshold of 3.5 [40], or an improvement of a factor of 2 in sensitivity. The distance reach of gravitational-wave detectors scales linearly with the inverse of SNR. Such potential improvement in gravitational-wave sensitivity, in a joint GW-neutrino search, will increase the science reach of the GW instruments relative to what they can achieve alone.

2) A gravitational-wave coincidence requirement also has the potential to improve the sensitivity of neutrino experiments by relaxing the criteria for detection. For example, Super-K's "distant" burst search [37] requires two neutrino events (with energy threshold $17 \mathrm{MeV}$ ) within 20 seconds, which corresponds to approximately $8 \%$ probability of detecting a supernova in Andromeda. The accidental coincidence rate for this criterion is less than one per year; the single event rate at this threshold is about 1 per day. If one could achieve an acceptable accidental rate by requiring coincidence of a single neutrino event with a gravitational-wave signal, then the probability of a core-collapse event in Andromeda satisfying the search criterion would be about $35 \%$. . Distant burst search parameters could be re-optimized with respect to current ones; the neutrino event energy threshold could potentially be reduced, further improving sensitivity.

\section{Conclusions}

The evolution of the gravitational wave detectors field is well defined: the laser interferometers are evolving toward their second generation: the advanced (Virgo and LIGO) detectors. According to the current gravitational wave sources modelling, when these apparatuses will reach their nominal sensitivity, the detection of the gravitational waves seems assured in few months of data taking. But the sensitivity needed to test the Einstein's gravity in strong field condition or to realize precision gravitational wave astronomy goes beyond the expected performances of the advanced detectors.

The fundamental limitations at low frequency of the sensitivity of the 2 nd generation detectors are given by the seismic noise, the related gravitational gradient noise (so-called Newtonian noise) and the thermal noise of the suspension of the test masses. To circumvent these limitations new infrastructures are necessary: an underground site for the detector, to limit the effect of the seismic noise, and, likely, cryogenic facilities to cool down the mirrors to directly reduce the thermal vibration of the test masses.

ET (Einstein Telescope http://www.et-gw.eu/) is a project for a future European third generation 
gravitational wave detector to be realized underground [43]. The realization of the ET research infrastructure, allowing operations for many decades, will be triggered by the first gravitational wave detection with the start of the site preparation beginning as early as 2018 and with scientific data being available in the following decade. Similarly, there will be the possibility for a high-sensitivity largebandwidth observatory to be built in other continents.

The low-frequency range, below $1 \mathrm{~Hz}$, includes a large and diverse population of strong gravitational wave sources that can only be observed at these frequencies. Detection technologies are diverse and range from polarization measurements of the cosmic microwave background and pulsar timing to spacecraft tracking and large baseline laser interferometry. All of these technologies will eventually be used to observe the complete gravitational wave spectrum covering more than 20 orders of magnitude in frequency. The scientific objectives of space-based and groundbased instruments are complementary in the same way that optical and x-ray astronomy are complementary and have provided information about different types of astrophysical objects and phenomena.

eLISA, a space-based interferometer will open the low-frequency gravitational wave window from $0.1 \mathrm{mHz}$ to $0.01 \mathrm{~Hz}$. eLISA is the gravitational wave community's highest priority for a space-based mission. The goal of a launch of eLISA in, say, 2025 is technologically feasible and entirely timely, considering that the technology precursor mission, LISA Pathfinder, will be launched in 2014.

Also worth to be mentioned are the growing efforts to utilize radio astronomy for the detection of gravitational waves in the nanohertz frequency band, with the formation of the International Pulsar Timing Array (IPTA) collaboration. These efforts are complementary to those of the ground- and spacebased laser interferometric projects and could well lead to observation of gravitational radiation in this band at the end of this decade.

\section{References}

[1] A. Einstein. Sitzungsberichte der Koniglich Preußischen Akademie der Wissenschaften (Berlin), page 688, (1916).

[2] P. Astone et al. (IGEC Collaboration). Phys. Rev. D 76 (10), 102001 (2007).

[3] P. Astone et al., Phys. Rev. Lett. 91 (11), 111101 (2003).

[4] S. Vitale et al., Phys. Rev. D 55, 1741 (1997).

[5] LIGO, GEO, VIRGO: http://ligo.caltech.edu, http://geo600.aei.mpg.de/, http://www.virgo.infn.it

[6] 3. K. Kuroda and LCGT Collaboration. Class. Quantum Grav., 27, 084004 (2010).

[7] B. Iyer et al. LIGO-India, Proposal of the Consortium for Indian Initiative in Gravitational-wave Observations (IndIGO). Technical report, LIGO Scientific Collaboration, 2011. Available at https://dcc.ligo.org/cgi-bin/DocDB/ShowDocument?docid=75988.

[8] P. R. Saulson. Fundamentals of Interferometric Gravitational Wave Detectors. World Scientific, Singapore (1994).

[9] K. S. Thorne. In S. W. Hawking and W. Israel, editors, 300 Years of Gravitation, Cambridge, UK, Cambridge University Press (1987).

[10] B. P. Abbott et al. (LIGO Scientific Collaboration). Phys. Rev. D 80 (10), 102001 (2009).

[11] J. Abbott et al. (LIGO Scientific Collaboration and Virgo Collaboration). Phys. Rev. D 81(10), 102001 (2010).

[12] M. Maggiore, Phys. Report 331, 283-367 (2000).

[13] M. Maggiore, VIR-NOT-PIS-1390-113 IFUP-TH 58/97, March 1998

[14] E.W. Kolb and M.S. Turner, The Early Universe, Addison Wesley (1990).

[15] V. Mandic and A. Buonanno, Phys. Rev. D73, 063008 (2006), arXiv:astro-ph/0510341.

[16] R. Apreda et al., Nucl. Phys. B631, 342 (2002), arXiv:gr-qc/0107033.

[17] B. Allen and J. D. Romano, Phys. Rev. D59, 102001 (1999), arXiv:gr-qc/9710117. 
[18] B. P. Abbott et al., Reports on Progress in Physics 72, 076901 (2009), arXiv:gr-qc/0711.3041.

[19] F. Acernese et al., Classical and Quantum Gravity 25, 114045 (2008).

[20] H. Grote for the LIGO Scientific Collaboration, Classical and Quantum Gravity 25, 114043 (2008).

[21] L. Grishchuk, JETP Letters 23, 293 (1976).

[22] B. P. Abbott et al., Nature 460, 990 (2009), arXiv:astro-ph/0910.5772.

[23] T. Regimbau and J. A. de Freitas Pacheco, Astron. Astrophys. 447, 1 (2006).

[24] V. Ferrari, S. Matarrese, and R. Schneider, MNRAS 303, 247 (1999), arXiv:astro-ph/9804259.

[25] X. Siemens, V. Mandic, and J. Creighton, Phys. Rev. Lett. 98, 111101 (2007).

[26] B. P. Abbott et al. Phys.Rev. D85 122001 (2012).

[27] N. K. Glendenning, Compact Stars, Springer-Verlag, Berlin, (2000).

[28] S. L. Shapiro and S. A. Teukolsky, Black Holes, White Dwarfs and Neutron Stars (Wiley, New York, 1983).

[29] E. Coccia, F. Dubath, M. Maggiore, Phys. Rev. D 70, 084010 (2004)

[30] K. S. Cheng and Z.G. Dai, Astrophys. J. 492, 281 (1998).

[31] G. F. Marranghello, C. A. Z. Vasconcellos, and J. A. de Freitas Pacheco, Phys. Rev. D 66, 064027 (2002).

[32] G. Miniutti et al., Mon. Not. R. Astron. Soc. 338, 389 (2003); Astrophys. J. 411, L33 (1993).

[33] N. Andersson, D. I. Jones, and K. D. Kokkotas, Mon. Not. R. Astron. Soc. 337, 1224 (2002).

[34] F. Woosley and H-T Janka arXiv:astro-ph/0601261

[35] I. Leonor et al., Class. Quant. Grav. 27, 084019 (2010)

[36] G. Pagliaroli, et al. Phys. Rev. Lett. 103, 031102, (2009).

[37] M Ikeda et al. (Super-KAMIOKANDE Collaboration). ApJ., 669, 519 (2007).

[38] N. Y. Agafonava et al. Astropart. Phys. 28, 516 (2008)

[39] L. Cadonati, F.P. Calaprice, and M.C. Chen, Astropart. Phys. 16 361, (2002)

[40] A. Piepke et al. Nucl. Phys. B 91, 99 (2001)

[41] P. Antonioli et al. New J. Phys. 6, 114 (2004)

[42] K. Scholberg, Astronomische Nachrichten 329, 337 (2008)

[43] B. Sathyaprakash et al., Class. Quant. Grav. 29, 084019 (2012) 